Evaluating the Risk Factors and Induced Cardiotoxicity in Breast

\title{
Cancer Patients
}

\author{
Samira Razaghi ${ }^{1}$, Sasan Razmjoo ${ }^{1}$, Mitra Ranjbaran ${ }^{2}$ and Habib Haybar (iD) ${ }^{2,}{ }^{*}$ \\ ${ }^{1}$ Department of Clinical Oncology, Ahvaz Jundishapur University of Medical Sciences, Ahvaz, Iran \\ ${ }^{2}$ Atherosclerosis Research Center, Ahvaz Jundishapur University of Medical Sciences, Ahvaz, Iran \\ "Corresponding author: Atherosclerosis Research Center, Ahvaz Jundishapur University of Medical Sciences, Ahvaz, Iran. Email: takamoolsk@yahoo.com \\ Received 2020 June 02; Revised 2020 September 30; Accepted 2020 October 03.
}

\begin{abstract}
Background: Breast cancer (BC) is one of the most common cancers among women. The survival of the BC patients is based on new treatment protocols. Cardiotoxicity is the most common side effect in these patients.

Methods: This was a descriptive study in which we selected our patients randomly among the BC patients and used questionnaires for data completion.66 BC patients whose disease were confirmed according to the histology and laboratory data taken to the oncology section of Ahvaz Golestan hospital between 2017-2018. Risk factors, history of the patients, and the function of the heart were evaluated 6 and 12 months after chemotherapy through echocardiography.

Result: Six months after chemotherapy, the follow-up patients in 6 indicated five significant risk factor included Age $(\mathrm{P}<0.03)$, history of heart disease $(\mathrm{P}<0.02)$, blood pressure $(\mathrm{P}<0.00)$, diabetes $(\mathrm{P}<0.00)$, and cholesterol $(\mathrm{P}<0.04)$, which confirm the dysregulation function of heart and indication of cardiotoxicity. In 12 months follow- up, the result indicated that only the history of heart disease was significantly correlated with cardiotoxicity $(\mathrm{P}<0.01)$.

Conclusion: Finally, the detection of risk factors in BC can introduce a prognostic factor when cardiotoxicity occurs simultaneously with the conduction of chemotherapy.
\end{abstract}

Keywords: Anthracycline, Chemotherapy, Breast Cancer, Cardiotoxicity, Risk Factors

\section{Background}

Breast cancer (BC) is one of the most common cancers among women, especially in these days $(1,2)$. The incidence of $\mathrm{BC}$ is about $24.6 \%$ of all cancers that happen in women (3). In the previous decades, although the prevalence of BC-related mortality was high among patients, the survival rate of patients would increase based on the recent diagnosis and treatment strategy (4-6). The main problem in BC treatment is cardiotoxicity derived chemotherapy. BC is a heterogeneous disease so it has a variety of clinical and histological symptoms among the patients, but proper treatment can be helpful in the management of the symptoms (7-9). Anthracyclines such as Adriamycin and danomycin are one of the most commonly used chemotherapy, which inhibit the progressive growth and metastasis $(10,11)$. Studies indicated that the use of these drugs can affect the cardiovascular system and increase cardiotoxicity. Anthracyclines increase the production of reactive oxygen species (ROS). ROS production impairs cardiomyocyte function and induced cardiotoxicity. Anthracycline also leads to car- diac cell apoptosis due to the production of inflammatory mediators, impaired calcium metabolism, mitochondrial function, and ATP production (12). Several risk factors such as smoking, old age, history of heart disease can increase the possibility of cardiotoxicity among BC patients under chemotherapy $(13,14)$. Therefore, the detection of risk factors in BC patients and related pathogenesis mechanisms in cardiotoxicity induced by chemotherapy agents can be useful in the management of heart disease in cancer patients. Cho et al. evaluated the incidence of cardiotoxicity in BC patients. They showed risk factors such as old age, metastasis, and concomitant use of doxorubicin and trustuzumab could increase the incidence of cardiotoxicity in cancer patients (15). Chung et al. demonstrated that cardiotoxicity could occur in patients treated with doxorubicin at $300 \mathrm{mg} / \mathrm{m}$ dosage (2) and higher (16).

\section{Objectives}

Several risk factors are known to be associated with the occurrence of cardiotoxicity in patients, finding the main 
risk factors in patients can be very helpful in designing preventive strategies and identifying high-risk patients. However, these risk factors are no fully identified in Iran. So, it can be an important approach to prevent the occurrence of cardiotoxicity in patients. The present study aimed to consider the relation between the risk factors and induced cardiotoxicity in $\mathrm{BC}$ patients hospitalized in Ahvaz Golestan hospital.

\section{Methods}

\subsection{Patients and Control Selection}

All the participants in this study ( $\mathrm{n}=66)$, confirmed between 2017 - 2018, went to the oncology section of Ahvaz Golestan hospital to receive chemotherapy treatment. Table 1 provides all demography data and clinical parameters related to patients.

\subsection{Inclusion Criteria}

Age $>20$ years, confirmed BC according to histology and laboratory symptoms, absence of underlying diseases, and availability of the patient's information.

\subsection{Exclusion Criteria}

Heart dysfunction in the early treatment stage (first time echo), congenital heart disease, use of drugs that effect on heart function, cancel of follow-up, unavailability of patient information, and underlying disease. The following formula was used to determine the sample size based on reference (17). Before launching the study, informed consent was obtained from each patient. This study was approved by the ethics committee of Ahvaz Jundishapur University of Medical Sciences (IR.AJUMS.REC.1397.053).

Sample size determination formula:

$n=\frac{p_{1}\left(100-p_{1}\right)+p_{2}\left(100-p_{2}\right)}{\left(p_{1}-p_{2}\right)^{2}}$

\subsection{Evaluation Cardiotoxicity}

Cardiotoxicity is determined according to the following criteria, including chest pain, heartbeat, acute myocardial infarction (AMI), heart failure (HF), and arrhythmia. The patients' follow-up 6 and 12 months after chemotherapy by echocardiography. Then, the data related to echocardiography was evaluated for the detection of cardiotoxicity by the cardiologist.

\begin{tabular}{|c|c|}
\hline & No. (\%) \\
\hline \multicolumn{2}{|l|}{ Age } \\
\hline $20-30$ & $0(0)$ \\
\hline $30-40$ & $9(13.6)$ \\
\hline $40-50$ & $27(40.9)$ \\
\hline$>50$ & $30(45.5)$ \\
\hline \multicolumn{2}{|c|}{ History of Smoking } \\
\hline Yes & $1(1.5)$ \\
\hline No & $65(98.5)$ \\
\hline \multicolumn{2}{|l|}{ BMI } \\
\hline$\geq 30$ & $19(28.8)$ \\
\hline$<30$ & $47(71.2)$ \\
\hline \multicolumn{2}{|c|}{ History of heart disease } \\
\hline Yes & $8(12.1)$ \\
\hline No & $58(87.9)$ \\
\hline \multicolumn{2}{|c|}{ History of Blood pressure } \\
\hline$\geq 135 / 85$ & $16(24.2)$ \\
\hline$<135 / 85$ & $50(75.8)$ \\
\hline \multicolumn{2}{|c|}{ History of Diabetes } \\
\hline $\mathrm{FBS} \geq 126$ & $14(21.2)$ \\
\hline FBS $<126$ & $52(78.8)$ \\
\hline \multicolumn{2}{|l|}{ HDL } \\
\hline$\geq 40$ & $55(83.3)$ \\
\hline$<40$ & $11(16.7)$ \\
\hline \multicolumn{2}{|l|}{ LDL } \\
\hline$\geq 100$ & $18(27.3)$ \\
\hline$<100$ & $48(72.7)$ \\
\hline \multicolumn{2}{|l|}{ TG } \\
\hline$\geq 150$ & $11(16.7)$ \\
\hline$<150$ & $55(83.3)$ \\
\hline \multicolumn{2}{|l|}{ Cholesterol } \\
\hline$\geq 200$ & $16(24.2)$ \\
\hline$<200$ & $50(75.8)$ \\
\hline \multicolumn{2}{|l|}{ Physical activity } \\
\hline Yes & $8(12.1)$ \\
\hline No & $58(87.9)$ \\
\hline \multicolumn{2}{|l|}{ CRP } \\
\hline$\geq 6$ & $5(7.6)$ \\
\hline$<6$ & $61(92.4)$ \\
\hline
\end{tabular}

Abbreviations: BMI, body mass index; BP, blood pressure; FBS, fast blood sugar; HDL, high-density lipoprotein; LDL, low-density lipoprotein; TG, triglyceride; CRP, C-reactive protein. 


\subsection{Risk Factors for Serious Cardiotoxicity}

To evaluate the association between risk factors and cardiotoxicity, we considered several parameters such as a history of smoking, high blood pressure, history of diabetes, history of heart disease, lipid profile, obesity, activity, and Body mass index (BMI). BMI was calculated as the bodyweight in kg by height in $\mathrm{m} 2$ (18) TG, CHOL, and HDL were also measured by colorimetric method using the laboratory kits of Pars Azmoon (Tehran, Iran). Also, the Friedewald formula was used for the calculation of LDL (19).

$$
\begin{aligned}
& L D L_{c}(m g / d L)=T C(m g / d L) \\
& -H D L_{c}(m g / d L)-T G(m g / d L) / 5
\end{aligned}
$$

\subsection{Statistical Analysis}

To describe the data, we used mean (SD) median, and midrange quartiles in quantitative variables; however, frequency and percentage were used in qualitative variables. For single-variable analysis of data, we used t testing (in case of normal data) and Mann-Whitney testing (in case of non-normal data). We performed all analyses using SPSS software, version 22 (SPSS, Inc.). $\mathrm{P}<05$ was considered to be statistically significant.

\section{Results}

\subsection{Association Between Risk Factors and Ejection Fraction in 6} Months After Treatment

To evaluate the correlation between risk factors and cardiotoxicity, we follow-up function of heart patients in 6 months' period by echocardiography. The result indicated statistical significant between several risk factors and heart dysfunction. Risk factors include age (P-Value: 0.03), history of heart disease (P-Value: 0.02), history of high blood pressure (P-Value: 0.00), diabetes (P-Value: $0.00)$, and cholesterol (P-Value: 0.040) (Table 2).

\subsection{Association Between Risk Factors and Ejection Fraction in 12 Months After Treatment}

In the second six- month follow-up, heart function was evaluated by echocardiography. The results indicated that the relationship between blood pressure and heart dysfunction was the most significant among the five risk factors investigated in the first six months of follow up (P-

\begin{tabular}{|c|c|c|c|}
\hline \multirow{2}{*}{ Risk Factor } & \multicolumn{2}{|c|}{$\operatorname{EF}(\mathrm{N})$} & \multirow{2}{*}{ P-Value } \\
\hline & $\geq \mathbf{5 0}$ & $<\mathbf{5 0}$ & \\
\hline Age & & & 0.03 \\
\hline $20-30$ & 0 & 0 & \\
\hline $30-40$ & 8 & 1 & \\
\hline $40-50$ & 25 & 2 & \\
\hline$>50$ & 20 & 10 & \\
\hline BMI & & & 0.86 \\
\hline$\geq 30$ & 15 & 4 & \\
\hline$<30$ & 38 & 9 & \\
\hline History of heart disease & & & 0.02 \\
\hline Yes & 4 & 4 & \\
\hline No & 49 & 9 & \\
\hline History of Blood pressure & & & 0.00 \\
\hline$\geq 135 / 85$ & 5 & 11 & \\
\hline$<135 / 85$ & 48 & 2 & \\
\hline History of Diabetes & & & 0.00 \\
\hline FBS $\geq 126$ & 7 & 7 & \\
\hline $\mathrm{FBS}<126$ & 45 & 8 & \\
\hline HDL & & & 0.12 \\
\hline$\geq 40$ & 7 & 4 & \\
\hline$<40$ & 45 & 9 & \\
\hline LDL & & & 0.08 \\
\hline$\geq 100$ & 12 & 6 & \\
\hline$<100$ & 41 & 7 & \\
\hline TG & & & 0.48 \\
\hline$\geq 150$ & 8 & 45 & \\
\hline$<150$ & 3 & 10 & \\
\hline Cholesterol & & & 0.04 \\
\hline$\geq 200$ & 10 & 6 & \\
\hline$<200$ & 43 & 7 & \\
\hline Physical activity & & & 0.68 \\
\hline Yes & 6 & 2 & \\
\hline No & 47 & 11 & \\
\hline CRP & & & 0.23 \\
\hline$\geq 6$ & 3 & 2 & \\
\hline$<6$ & 50 & 11 & \\
\hline
\end{tabular}
Value: 0.01) (Table 3 and Figure 1).
Abbreviations: BMI, body mass index; EF, eject fraction; BP, blood pressure; FBS, fast blood sugar; HDL, high-density lipoprotein; LDL, low-density lipoprotein; TG, triglyceride; CRP, C-reactive protein. 

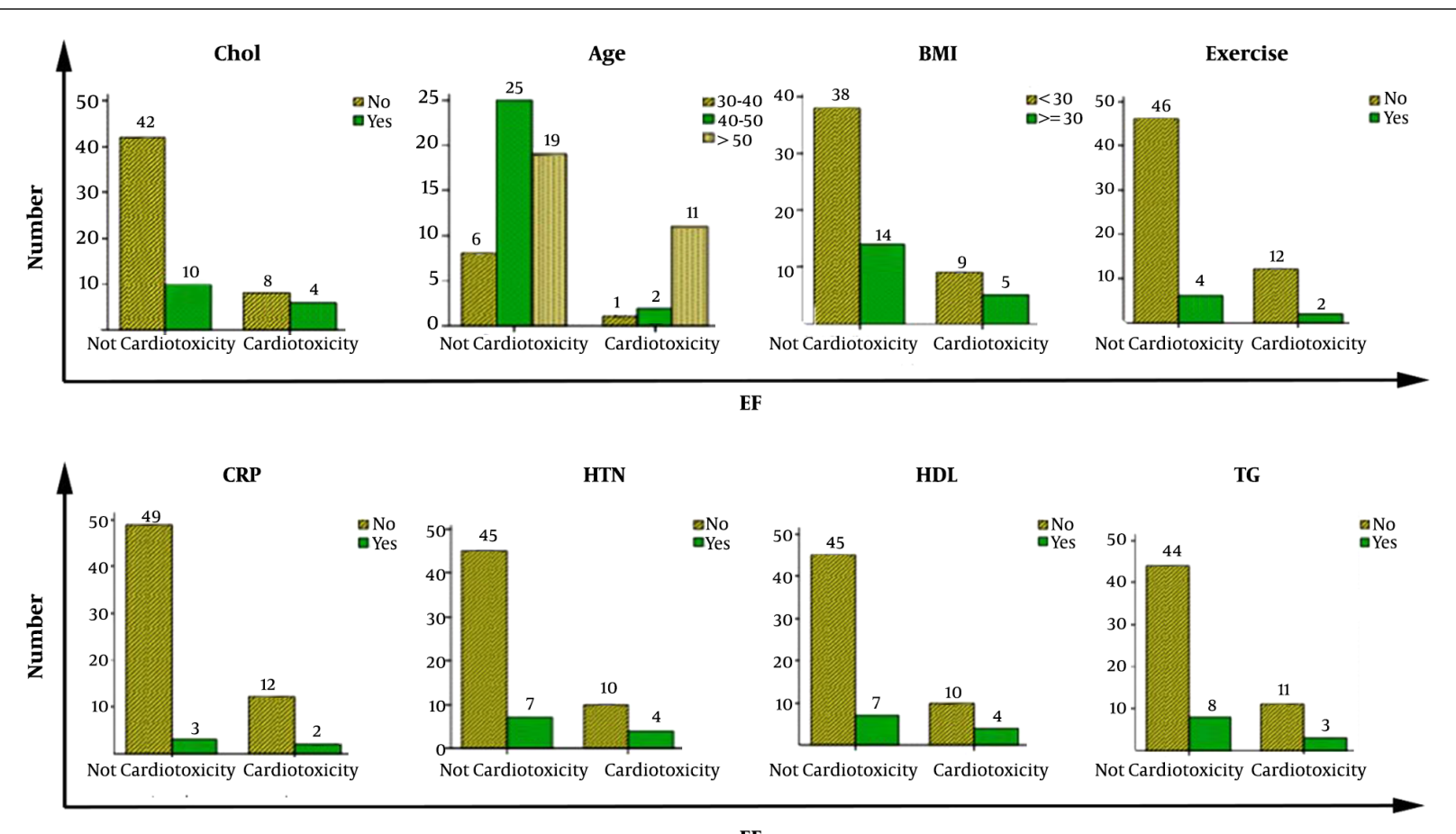

EF

Figure 1. Evaluation of risk factors in patients during 12 moths fallow up.

\section{Discussion}

$\mathrm{BC}$ is heterogeneous and has many risk factors involved in its incidence and progression. Studies have shown that many inherited factors such as family history and the occurrence of mutations and acquired factors are essential to play a role in the progression of the disease (20). Today, $\mathrm{BC}$ is one of the main reasons of mortality in the world; however, based on the recent therapeutic strategies, the survival of patients were increased $(21,22)$. Chemotherapyinduced cardiotoxicity is an important problem in cancer treatment $(23,24)$. The result of this study indicates that cardiotoxicity in cancer patients is associated with several risk factors, including disease history, life style (25). Meredith et al. evaluated the association of the risk factors and anthracyline in pathogenesis cardiomyopathy of acute myeloid leukemia (AML). They indicated that there was no statistical significance among the history of disease (P-Value: 0.4), BMI (P-Value: 0.5), smoking (P-value: 0.9), and blood pressure (P-Value: 0.3 ) with cardiotoxicity. However, the results indicated that heart dysfunction is less frequent in AML patients who received low dosage anthracycline than the solid tumor patients (26). Kosalka et al. evaluated risk factors such as obesity, dyslipidemia, and diabetes in trastuzumab-induced cardiotoxicity in BC pa- tients. The result indicated that these risk factors could increase the possibility of cardiotoxicity in BC patients (27). Howden et al. showed the effectiveness of exercise on heart function after chemotherapy in $\mathrm{BC}$ patients receiving anthracycline. The results indicated that cardiotoxicity is less common in patients who has more exercise before chemotherapy (28). Anber et al. assessed the C-reactive protein (CRP) as a biomarker for detecting cardiotoxicity in $\mathrm{BC}$ patients, the result indicated no change in the level of CRP before and after the treatment. Therefore, this factor cannot be used as a biomarker in the detection of cardiotoxicity in BC patients (29). Doyle et al. indicated the presence of cardiovascular disease and heart failure in $\mathrm{BC}$ patients receiving chemotherapy could increase the incidence of cardiotoxicity compared with other cases (30). Macgregor et al. showed high blood pressure and cardiovascular disease could increase the incidence of cardiotoxicity in patients who receive chemotherapy (17). In this study, we evaluated the risk factors in inducing cardiotoxicity in $\mathrm{BC}$ patients; we followed up heart function in patients after 6 and 12 months of treatment by echocardiography. Risk factors were investigated and listed in Table 1. Six months after chemotherapy, the result showed 5 risk factors, including age (P-Value: 0.03), history of heart disease (P-Value: 


\begin{tabular}{|c|c|c|c|}
\hline \multirow{2}{*}{ Risk Factor } & \multicolumn{2}{|c|}{$\mathbf{E F}(\mathbf{N})$} & \multirow{3}{*}{$\begin{array}{c}\text { P-Value } \\
0.07\end{array}$} \\
\hline & \multirow{3}{*}{$\begin{array}{c}\geq \mathbf{5 0} \\
0\end{array}$} & \multirow{2}{*}{$<\mathbf{5 0}$} & \\
\hline Age & & & \\
\hline $20-30$ & & 0 & \\
\hline $30-40$ & 9 & 0 & \\
\hline $40-50$ & 27 & 0 & \\
\hline$>50$ & 25 & 5 & \\
\hline BMI & & & 0.30 \\
\hline$\geq 30$ & 17 & 2 & \\
\hline$<30$ & 45 & 2 & \\
\hline History of heart disease & & & 0.01 \\
\hline Yes & 7 & 2 & \\
\hline No & 55 & 2 & \\
\hline History of Blood pressure & & & 0.18 \\
\hline$\geq 135 / 85$ & 14 & 2 & \\
\hline$<135 / 85$ & 48 & 2 & \\
\hline History of Diabetes & & & 0.15 \\
\hline$\geq 126$ & 13 & 2 & \\
\hline$<126$ & 49 & 2 & \\
\hline HDL & & & 0.65 \\
\hline$\geq 40$ & 10 & 1 & \\
\hline$<40$ & 52 & 3 & \\
\hline LDL & & & 0.30 \\
\hline$\geq 100$ & 16 & 2 & \\
\hline$<100$ & 46 & 2 & \\
\hline TG & & & 0.65 \\
\hline$\geq 150$ & 11 & 1 & \\
\hline$<150$ & 51 & 3 & \\
\hline Cholesterol & & & 0.22 \\
\hline$\geq 200$ & 14 & 2 & \\
\hline$<200$ & 48 & 2 & \\
\hline Physical activity & & & 0.43 \\
\hline Yes & 8 & 0 & \\
\hline No & 54 & 4 & \\
\hline CRP & & & 0.10 \\
\hline$\geq 6$ & 4 & 1 & \\
\hline$<6$ & 58 & 3 & \\
\hline
\end{tabular}

Abbreviations: BMI, body mass index; EF, eject fraction; BP, blood pressure; FBS, fast blood sugar; HDL, high-density lipoprotein; LDL, low-density lipoprotein $\mathrm{TG}$, triglyceride; CRP, C-reactive protein.

0.02), history of blood pressure (P-Value: 0.00), diabetes (PValue: 0.00) and cholesterol (P-Value: 0.04) statistical sig- nificance with induced cardiotoxicity(Table 2). However, 12 months' follow-up indicated only the history of heart disease was statistically significant. (P-Value: 0.01) (Table 3).

\subsection{Conclusion}

Finally, chemotherapy can affect many organs, and the most affected organ is the cardiovascular system in BC patients. According to this study, some risk factors that induce cardiotoxicity means make the patient vulnerable to cardiotoxicity during the chemotherapy. So, detecting these factors can be used as a prognostic factor for assessing the situation of the patient before and after treatment.

\section{Acknowledgments}

This work was financially supported by grant CVRC9702 from the vice chancellor for research affairs of Ahvaz Jundishapur University of Medical Sciences. This paper was issued from the thesis of Mitra Ranjbran.

\section{Footnotes}

Authors' Contribution: H.H. has conceived the manuscript and revised it. S.R, S.R, and M.R wrote the manuscript.

Conflict of Interests: No conflicts of interest declared.

Ethical Approval: All the procedures performed in the studies involving human participants were in accordance with ethical standards of the local ethics committee of Ahvaz Jundishapur University of Medical Sciences (IR.AJUMS.REC.1397.053), as well as the 1964 Helsinki declaration.

Funding/Support: This work was financially supported by grant CVRC-9702 from the vice chancellor for research affairs of Ahvaz Jundishapur University of Medical Sciences.

Informed Consent: Written informed consent was obtained from all patients and healthy subjects.

\section{References}

1. Chantalat E, Boudou F, Laurell H, Palierne G, Houtman R, Melchers D, et al. The AF-1-deficient estrogen receptor ERalpha46 isoform is frequently expressed in human breast tumors. Breast Cancer Res. 2016;18(1):123. doi: 10.1186/s13058-016-0780-7. [PubMed: 27927249]. [PubMed Central: PMC5142410].

2. Ansari N, Shahrabi S, Khosravi A, Shirzad R, Rezaeean H. Prognostic significance of CHEK2 mutation in progression of breast cancer. Lab Med. 2019;50(3):e36-41. doi: 10.1093/labmed/lmz009. [PubMed: 31220302]. 
3. Sayad S, Ahmadi S, Nekouian R, Panahi M, Anbari K. Epidemiological and pathological characteristics of post-surgical cases of invasive breast cancer among ethnicities of Iran in 2018: A single center cross-sectional study. Arch. Oncol. 2020;26(1):6-9. doi: 10.2298/aoo190626007s.

4. Abdel-Razaq W, Alzahrani M, Al Yami M, Almugibl F, Almotham M, Alregaibah R. Risk factors associated with trastuzumab-induced cardiotoxicity in patients with human epidermal growth factor receptor 2-positive breast cancer. J Pharm Bioallied Sci. 2019;11(4):348-54. doi: 10.4103/jpbs.JPBS_276_18. [PubMed: 31619917]. [PubMed Central: PMC6791087]

5. Zayeri Z, Haybar H, Jalali MT, Zibara K. Mechanisms and biomarkers to detect chemotherapy-induced cardiotoxicity. Clin Cancer Investig J. 2017;6(5):207.doi:10.4103/ccij.ccij_47_17.

6. Javaherforooshzadeh F, Monajemzadeh SA, Soltanzadeh M, Janatmakan F, Salari A, Saeed H. A comparative study of the amount of bleeding and hemodynamic changes between dexmedetomidine infusion and remifentanil infusion for controlled hypotensive anesthesia in lumbar discopathy surgery: A double-blind, randomized, clinical trial. Anesthesiol Pain Med. 2018;8(2). doi:10.5812/aapm.66959.

7. Baselga J. Clinical trials of Herceptin (trastuzumab). Eur J Cancer. 2001;37 Suppl 1:S18-24. [PubMed: 11167087].

8. Baselga J, Bradbury I, Eidtmann H, Di Cosimo S, de Azambuja E, Aura C, et al. Lapatinib with trastuzumab for HER2-positive early breast cancer (NeoALTTO): a randomised, open-label, multicentre, phase 3 trial. Lancet. 2012;379(9816):633-40. doi:10.1016/S0140-6736(11)61847-3. [PubMed: 22257673]. [PubMed Central: PMC5705192].

9. Javaherforooshzadeh F, Amirpour I, Janatmakan F, Soltanzadeh M. Comparison of effects of melatonin and gabapentin on post operative anxiety and pain in lumbar spine surgery: A randomized clinical trial. Anesthesiol Pain Med. 2018;8(3). doi: 10.5812/aapm.68763.

10. Skeel R, Lachant N. Antineoplastic drugs and biologic response modifiers. Philadelphia: Lippincott Williams \& wilkins publisher; 2005.

11. Haybar H, Shahrabi S, Deris Zayeri Z, Pezeshki S. Strategies to increase cardioprotection through cardioprotective chemokines in chemotherapy-induced cardiotoxicity. Int J Cardiol. 2018;269:276-82. doi: 10.1016/j.ijcard.2018.07.087. [PubMed: 30054148].

12. Sobczuk P, Czerwinska M, Kleibert M, Cudnoch-Jedrzejewska A Anthracycline-induced cardiotoxicity and renin-angiotensinaldosterone system-from molecular mechanisms to therapeutic applications. Heart Fail Rev. 2020. doi: 10.1007/s10741-020-09977-1. [PubMed: 32472524].

13. Bansal N, Amdani S, Lipshultz ER, Lipshultz SE. Chemotherapyinduced cardiotoxicity in children. Expert Opin Drug Metab Toxicol. 2017;13(8):817-32. doi: 10.1080/17425255.2017.1351547. [PubMed: 28679288].

14. Serrano C, Cortes J, De Mattos-Arruda L, Bellet M, Gomez P, Saura C, et al. Trastuzumab-related cardiotoxicity in the elderly: a role for cardiovascular risk factors. Ann Oncol. 2012;23(4):897-902. doi: 10.1093/annonc/mdr348. [PubMed: 21828361].

15. Cho H, Lee S, Sim SH, Park IH, Lee KS, Kwak MH, et al. Cumulative incidence of chemotherapy-induced cardiotoxicity during a 2year follow-up period in breast cancer patients. Breast Cancer Res Treat. 2020;182(2):333-43. doi: 10.1007/s10549-020-05703-5. [PubMed: 32468335].

16. Chung WB, Yi JE, Jin JY, Choi YS, Park CS, Park WC, et al. Early cardiac function monitoring for detection of subclinical Doxorubicin cardiotoxicity in young adult patients with breast cancer.J Breast Cancer. 2013;16(2):178-83. doi: 10.4048/jbc.2013.16.2.178. [PubMed: 23843850]. [PubMed Central: PMC3706863].

17. Chavez-MacGregor M, Zhang N, Buchholz TA, Zhang Y, Niu J, Elting L, et al. Trastuzumab-related cardiotoxicity among older patients with breast cancer. J Clin Oncol. 2013;31(33):4222-8. doi:
10.1200/JCO.2013.48.7884. [PubMed: 24127446]. [PubMed Central: PMC3821011].

18. Zare Javid A, Hosseini SA, Gholinezhad H, Moradi L, Haghighi-Zadeh $\mathrm{MH}$, Bazyar H. Antioxidant and anti-inflammatory properties of melatonin in patients with type 2 diabetes mellitus with periodontal disease under non-surgical periodontal therapy: A double-blind, placebo-controlled trial. Diabetes Metab Syndr Obes. 2020;13:753-61. doi: 10.2147/DMSO.S242208. [PubMed: 32256092]. [PubMed Central: PMC7090208].

19. Gholinezhad H, Bazyar H, Rashidi H, Salehi P, Haghighi-zadeh $\mathrm{MH}$ Zare Javid A. Using ginger supplement in adjunct with non-surgical periodontal therapy improves metabolic and periodontal parameters in patients with type 2 diabetes mellitus and chronic periodontitis: A double-blind, placebo-controlled trial. J Herb Med 2020;20:100315. doi:10.1016/j.hermed.2019.100315.

20. Eastlack SC, Alahari SK. MicroRNA and breast cancer: Understanding pathogenesis, improving management. Noncoding RNA. 2015;1(1):1743. doi: 10.3390/ncrna1010017. [PubMed: 29861413]. [PubMed Central: PMC5932537].

21. Li Z, Chen Y, Yang Y, Yu Y, Zhang Y, Zhu D, et al. Recent advances in nanomaterials-based chemo-photothermal combination therapy for improving cancer treatment. Front Bioeng Biotechnol. 2019;7:293. doi: 10.3389/fbioe.2019.00293. [PubMed: 31696114]. [PubMed Central: PMC6817476].

22. You YH, Lin YF, Nirosha B, Chang HT, Huang YF. Polydopamine-coated gold nanostar for combined antitumor and antiangiogenic therapy in multidrug-resistant breast cancer. Nanotheranostics. 2019;3(3):26683. doi: 10.7150/ntno.36842. [PubMed: 31263658]. [PubMed Central: PMC6584135].

23. Kerkela R, Grazette L, Yacobi R, Iliescu C, Patten R, Beahm C, et al. Cardiotoxicity of the cancer therapeutic agent imatinib mesylate. Nat Med. 2006;12(8):908-16. doi: 10.1038/nm1446. [PubMed: 16862153]

24. Haybar H, Shahrabi S, Rezaeeyan H, Jodat H, Saki N. Strategies to inhibit arsenic trioxide-induced cardiotoxicity in acute promyelocytic leukemia. J Cell Physiol. 2019. doi: 10.1002/jcp.28292. [PubMed: 30770558].

25. Jensen SA, Sorensen JB. Risk factors and prevention of cardiotoxicity induced by 5 -fluorouracil or capecitabine. Cancer Chemother Pharmacol. 2006;58(4):487-93. doi: 10.1007/s00280-005-0178-1. [PubMed: 16418875].

26. Mort MK, Sen JM, Morris AL, DeGregory KA, McLoughlin EM, Mort JF, et al. Evaluation of cardiomyopathy in acute myeloid leukemia patients treated with anthracyclines. J Oncol Pharm Pract. 2020;26(3):680-7. doi: 10.1177/1078155219873014. [PubMed: 31500517].

27. Kosalka P, Johnson C, Turek M, Sulpher J, Law A, Botros J, et al. Effect of obesity, dyslipidemia, and diabetes on trastuzumabrelated cardiotoxicity in breast cancer. Curr Oncol. 2019;26(3):e31421. doi: 10.3747/co.26.4823. [PubMed: 31285674]. [PubMed Central: PMC6588065]

28. Howden EJ, Bigaran A, Beaudry R, Fraser S, Selig S, Foulkes S, et al. Exercise as a diagnostic and therapeutic tool for the prevention of cardiovascular dysfunction in breast cancer patients. Eur J Prev Cardiol. 2019;26(3):305-15. doi: 10.1177/2047487318811181. [PubMed: 30376366].

29. Anber ZNH, Saleh BOM, Al-Rawi SA. The cardiotoxicity effect of different chemotherapeutic regimens in Iraqi patients with breast cancer: A follow up study. Heliyon. 2019;5(8). e02194. doi: 10.1016/j.heliyon.2019.e02194. [PubMed:31406940]. [PubMed Central: PMC6684458].

30. Doyle JJ, Neugut AI, Jacobson JS, Grann VR, Hershman DL. Chemotherapy and cardiotoxicity in older breast cancer patients: a population-based study. J Clin Oncol. 2005;23(34):8597-605. doi: 10.1200/JCO.2005.02.5841. [PubMed: 16314622]. 